\title{
Agustín Sánchez Rodrigo y la epigrafía en Serradilla, Cáceres, y sus alrededores
}

\section{Agustín Sánchez Rodrigo and the inscriptions from Serradilla and environs, Cáceres, Spain}

\author{
José-VIDAL MADRUGA* \\ Joaquín L. GómEZ-PANTOJA**
}

\begin{abstract}
RESUMEN
ABSTRACT

Este artículo examina la correspondencia entre Fidel Fita, prestigioso académico de

la Historia, y Agustín Sánchez Rodrigo, vecino de Serradilla, Cáceres, cuyo objeto fue la media docena de inscripciones antiguas de cuya existencia en su pueblo y alrededores el segundo había tenido noticias.

This paper assesses the epistolar exchange between Fidel Fita, a leading member of the Spanish Real Academía de la Historia, and Agustín Sánchez Rodrigo, the editor of a local newspaper in Serradilla, province of Cáceres, Spain. The motive behind the correspondence was Sánchez' interest in sharing several ancient inscripcions found in Serradilla and environs.
\end{abstract}

\author{
PALABRAS CLAVE \\ Epigrafía, Lusitania, Historiografía.
}

KEYWORDS

Epigraphy, Roman Spain, Historiography

Recibido el 3 de septiembre de 2012. Aceptado el 11 de septiembre de 2012

\section{UN INDIVIDUO SINGULAR}

Agustín Sánchez Rodrigo nació en Serradilla en 1870, en el seno de una familia de rentistas acomodados y con inquietudes intelectuales ${ }^{1}$. Desde muy joven

*Archivo Epigráfico de Hispania, pepomad@gmail.com

" Dpto. de Historia, Universidad de Alcalá / Archivo Epigráfico de Hispania, gomez.pantoja @uah.es. Este trabajo es consecuencia directa de la gestión y mantenimiento de la base de datos epigráfica de acceso libre Hispania Epigraphica Online (http://www.eda-bea.es), que se financia con los fondos públicos del proyecto HAR2011-29108-C04-02: «El taller de ORDO: Representaciones gráficas de la influencia y el poder de las élites en la pars Occidentalis del Imperio Romano". Agradecemos a Carlos Bravo (www.serradilla.com) que nos facilitase acceso a la colección completa de «El Cronista».

${ }^{1}$ No hay demasiada información impresa sobre el personaje: además de los títulos citados en páginas siguientes, vid. la biografía completa, pero novelada, de CHAMORRO, Víctor, Sin Raíces, Plasencia, $2^{\mathrm{a}}$ ed., Sánchez Rodrigo, 1970; También CAJIDE LABRADOR, Juan José, Extremadura : his- 
mostró una notable inclinación por eso que ahora se llama «animación cultural»y, mezclando a partes iguales entusiasmo con ingenuidad, se le recuerda en su pueblo como fundador de una sociedad cultural, de un semanario y otras publicaciones periódicas, y antes de eso, cuando apenas tenía 16 años, de una libreria. Estas iniciativas apuntan a un individuo de buena posición, ilustrado, autodidacta y simpatizante en mayor o menor medida de la corriente intelectual denominada «regeneracionista» que, en el tránsito del siglo XIX al XX, sostenía que la decadencia española era primordialmente un problema de egoísmo social y ausencia de la noción de bien común, menosprecio de los valores propios y de la tradición y sobre todo, incultura, que impedía un aprovechamiento eficiente de los recursos disponibles y, en consecuencia, el aumento individual y colectivo de la riqueza. Muchos de esos principios se expresaron de forma literaria, pero hubo también quien quiso corregirlos en la práctica, dejándose llevar por el entusiasmo y sin consider las dificultades reales. Aunque a Sánchez Rodrigo no le faltó la inclinación erudito-literaria $^{2}$, es claro que su personalidad correspondía al segundo tipo, el del reformista con medios que ingenuamente creía que bastaba esfuerzo y un poco de dinero para cambiar las cosas. Lógicamente, los proyectos de Sánchez Rodrigo terminaron en fracaso inmediato o perecieron por consunción: en primer lugar, Serradilla se encuentra situada en medio de una penillanura tan masiva y agreste que resulta más fácil circundarla que atravesarla; sólo el río Tajo ha sido capaz de pasar por medio de ella, pero encajonándose tan profundamente que su cruce se convierte en el más difícil obstáculo a la viabilidad; todavía hoy, los principales caminos y carreteras de la comarca —el ferrocarril incluido- marginan al pueblo y sus habitantes llevan un siglo quejándose de ello. Y luego, porque el pueblo está rodeado de buena tierra, capaz de sostener un número relativamente alto de habitantes, que siguió creciendo hasta bien entrado el pasado siglo; pero por la época en la que Sánchez Rodrigo decidió transformar la realidad cultural de su aldea, se estima que el $56 \%$ de los varones y el $82 \%$ de sus vecinas eran analfabetos ${ }^{3}$.

El único perjudicado de estas iniciativas fue el bolsillo de su promotor, lo que no impidió que en 1905 se embarcara en una gran empresa comercial que es responsable, en última instancia, de este artículo. El año anterior, un paisano y amigo, Maestro Nacional, le dio a conocer un método de aprendizaje lecto-escritor con el que llevaba experimentando en sus clases y al que llamaba Rayas, por basarse en sencillos dibujos que el alumno debía aprender a copiar antes de hacerlo con las

torias de la utopía, Badajoz, Carisma, 1996. Finalmente, la Unión de Bibliofilos Extremeños le dedicó monográficamente las «XIII Jornadas Bibliográficas Bartolomé J. Gallardo» de 2006, vid. Actas de las las XIII y XIIII Jornadas Bibliográficas Bartolomé J. Gallardo, Badajoz, Unión de Bibliofilos Extremeños, 2009.

2 Aparte de los sueltos y noticias publicados en sus primeras y fallidas empresas periodísticas y los impresos en El Cronista durante los 17 años de actividad del quincenario, Sánchez Rodrigo fue autor de dos libros: el primero, Un año de vida serradillana, Serradilla, El Cronista, 2 ed. 1915 y 1918, es una recopilación de artículos y noticias aparecidos en El Cronista; el otro, Nociones y tablas de Aritmética, Serradilla, s.a, parece un auxilio docente con largo recorrido editorial, pues consta una edición de 1972.

3 Véase la serie histórica de censos demográficos del Instituto Nacional de Estadística en http://www.ine.es/inebaseweb/71807.do?language=0. Tanto éste como los demás URL que se citan seguidamente estaban activos al tiempo de la redacción del artículo. Sobre el analfabetismo, VILANOVA RIBAS, Mercedes y MORENO JULIÁ, Xávier, Atlas de la evolución del analfabetismo en España de 1887 a 1981, Madrid, MEC, 1992 pág. 234. 
letras ${ }^{4}$. Cuando el propio autor y sus allegados más cercanos dudaron de que se pudiera dar a conocer, e implantar el nuevo método, Sánchez Rodrigo se ofreció a editarlo y distribuirlo, en el espíritu que antes se ha descrito. De nuevo a su costa, Sánchez Rodrigo encargó en Madrid la tipografía adecuada, los galvanos y la tirada de la primera edición del Rayas, que fueron enviados por tren a la estación más cercana y desde allí, transportados a lomos de caballería el resto del camino hasta Serradilla, ya que «el plan de negocio» del editor incluía difundir, distribuir y vender el método desde su pueblo.

Sirviéndose de la rapidez del sistema postal, Sánchez Rodrigo agotó la primera edición del Rayas como publicidad, enviando ejemplares a los maestros de toda España. En 1909, decidió abaratar costes encargando de nuevo la impresión en Madrid pero realizando el manipulado final de los cuadernos en Serradilla, lo que requirió adquirir máquinas adecuadas; la tendencia a la autosuficiencia se acrecentó aún más cuando empezaron a recibirse los primeros pedidos del Rayas: tras adquirir una minerva a pedal de formato folio, que llegó al pueblo por el medio habitual de transporte, desguazada, sin montador ni instrucciones para armarla, la tercera edición del método se tiró íntegramente en Serradilla, de tal modo que Sánchez Rodrigo - que había instalado en 1912 un estudio de fotografía- es considerado el primer editor comercial moderno de Extremadura. Hasta ese momento, lo gastado en la edición y difusión del método Rayas superaba las 50.000 pesetas, una considerable cantidad para la época, y que se había obtenido de las rentas propias y de préstamos. Sin embargo, la inversión no había producido aún sus frutos, a pesar de que el método se había implantado en algunas escuelas, recibido premios en distintas exposiciones y su utilidad era unánimemente reconocida ${ }^{5}$.

Para difundir aún más el método lecto-escritor sin incrementar costes, Agustín Sánchez decidió aprovechar que las tarifas postales para periódicos eran más baratas que las de las cartas, y a comienzos de 1916 fundó El Cronista, una revista quincenal destinada al Magisterio Nacional -y por lo tanto, un adecuado soporte publicitario del Rayas-, pero que contenía también noticias y escritos de interés para Serradilla y su comarca ${ }^{6}$. La publicación estuvo activa hasta diciembre de 1932 , editándose 407 números y un suplemento pedagógico, Educación y Enseñanza?. Sánchez Rodrigo murió tras una operación quirúrgica en febrero de 1933, aún en dificultades económica, pero cuando el Rayas comenzaba a ser universalmente co-

4 SÁNCHEZ MATEOS, Iván, «La verdad sobre el Método Rayas», en http://elavisadordebadajoz.zoomblog.com/archivo/2011/12/03/la-verdad-sobre-el-Metodo-Rayas_3.html.

5 BARBERO MATEOS, Jesús, «Cien años de una innovación sin precedentes. El Rayas», en XXXII Coloquios Históricos de Extremadura, Trujillo, 2003, cuya versión digital está disponible en: http://chde.org/index.php?option=com_content\&view=article\&id=237:innovacion-sin-precedentes-el- rayas \& catid $=33: 2003 \&$ Itemid $=50$.

6 CALDERÓN ESPAÑA, M. Concepción y REAL APOLO, Carmelo, «Educación y sociedad en el periódico «El Cronista» de Serradilla (Cáceres) (1916-1932)», en Hermoso Ruiz, Faustino (ed.), VIII Congreso de Extudios Extremeños, Badajoz, Diputación, 2007, pág. 1563-1579.

7 BARBERO MATEOS, Jesús, «Educación y Enseñanza. Un suplemento educativo monográfico en los albores del siglo XX», Coloquios Históricos de Extremadura, 2004; Edición digital en http://www.chde.org/index.php?option=com_content\&view=article\&id=196:educacion-y-ensenanza-albores-siglo-xx\&catid=32:2004\&Itemid=41. 
nocido y apreciado; después de la Guerra Civil, sus sucesores trasladaron la editorial a la mejor comunicada Plasencia y siguieron produciendo y difundiendo el producto hasta 1975, vendiendo en total más de 52 millones de ejemplares.

\section{SERRADILLA EN LA ACADEMIA DE LA HISTORIA}

Por su exitosa carrera en la Academia de la Historia -miembro Correspondiente desde 1865, individuo de Número en 1877, Anticuario Perpetuo (19091913) y Director del docto Cuerpo desde 1912 (interino) hasta su muerte en 1918-, el Padre Fidel Fita Colomer, S.J. es sin duda, uno de los personajes más influyentes de la historiografía española en las décadas inmediatas al tránsito entre los siglos XIX y $X^{8}$. Su importancia, además, la incrementa una extensa producción literaria, que abarcó primordialmente la edición de fuentes documentales de diversas épocas y temáticas. Fita fue un polígrafo, interesado tanto en los más antiguos testimonios de las lenguas paleo-hispanas como en la música medieval, en la judería hispana como en el culto a los mártires, y que encontró en el Boletín de la Real Academia de la Historia (del que fue director desde 1883 hasta su muerte), una segura y prestigiosa plataforma de difusión para sus escritos, Ilegando a publicar más de 600 piezas en ese medio. Muchas de ellas tienen que ver con cuestiones epigráficas, a las que Fita prestó mucha atención por motivos coyunturales — los trabajos contemporáneos de Emil Hübner catalogando las inscripciones latinas peninsulares-, pero también por razones de índole ideológica ya que, como buen historicista, consideraba que la averiguación del más remoto pasado de un pueblo reposaba últimamente en el conocimiento de su lengua ${ }^{9}$.

La magnitud de lo que Fita hizo en este campo ha podido fácilmente minusvalorarse porque, tratándose de un continuo goteo de noticias a lo largo de más de cuarenta años, es difícil valorar; pero el catálogo de Mauleón ${ }^{10}$ hace justicia al esfuerzo: entre 1887 y 1948, aparecieron en el Boletín de la Academia de la Historia más de dos mil noticias de inscripciones antiguas; todas, salvo unas 70, tienen fecha de publicación anterior a 1919. Tampoco son todos esos informes de la autoría del docto jesuita, pero sí la inmensa mayoría ya que, al asumir la dirección del Boletín académico, recayó sobre él la responsabilidad de producir, imprimir y distribuir puntual-

8 Su résumé en la Academia en RUMEU DE ARMAS, Antonio, La Real Academia de la Historia, Madrid, Real Academia de la Historia, 2001, págs. 54, 56 y 164. Pero resulta más informativa y útil la compacta biografía literaria e institucional de PASAMAR ALZURIA, Gonzalo y PEIRÓ MARTíN, Ignacio, Diccionario Akal de historiadores españoles contemporáneos (1840-1980), Tres Cantos (Madrid), Akal, 2002, pág, 254-256.

9 Por circunstancias peculiares, el anticuarismo en general y la epigrafía en particular han sido los aspectos más estudiados de la actividad intelectual del académico, vid. ABASCAL PALAZÓN, Juan Manuel, Fidel Fita (1835-1918). Su legado documental en la Real Academia de la Historia, Madrid, 1999. Pero, tratándose de un reputado representante del historicismo de la Restauración, falta un análisis de sus influencias, de su ideología y de los motivos tras sus escritos.

10 Vid. MAULEÓN, María Dolores, Indices de las inscripciones latinas publicadas en el Boletín de la Real Academia de la Historia (1877-1950), Pamplona, Universidad de Navarra, 1983. 
mente el órgano oficial de la Academia de la Historia. En la tesitura de tener que cuadrar el número de pliegos previstos para cada fascículo, Fita recurrió a la receta universal de los editores que, es bien sabido, sólo precisa dos componentes, la imperiosa necesidad y la falta de tiempo. Fita completó frecuentemente con piezas de su propio puño y letra las páginas en falta y la materia para esos sueltos salió con frecuencia de la correspondencia sobre asuntos anticuarios - generalmente hallazgos epigráficos - que una plétora de corresponsales de toda España enviaban a la Academia, aunque cada vez se hizo más frecuente que esa cartas estuvieran dirigidas directamente al propio jesuita. La constancia en el empeño, el número y variedad de las lápidas publicadas y la puntualidad y autoridad del Boletín de la R.A.H, atribuyeron a Fita una considerable autoridad, que se vio respaldada por las fluidas relaciones con $\mathrm{E}$. Hübner, que frecuentemente se sirvió de las informaciones y los corresponsales del académico para llevar a cabo la revisión y actualización del volumen II del Corpus Inscriptionum Latinarum. A pesar del tiempo transcurrido, aún se considera a Fita una fuente válida para el estudio de las inscripciones antiguas de Hispania, especialmente cuando se trata de epígrafes publicados por él que ya no pueden revisarse, porque se destruyeron o porque se desconoce su paradero.

Gracias a que en años recientes ha sido posible la catalogación de los muchos papeles personales encontrados a su muerte en la celda de la madrileña Casa Profesa de la Compañía de Jesús ${ }^{11}$, ahora sabemos que Fita dio a la imprenta sólo una parte de la correspondencia recibida, permaneciendo el resto de las cartas inéditas. Estas contienen información miscelánea sobre las circunstancias de los hallazgos, sus medidas. su paradero y frecuentemente adjuntaban imágenes, al principio, dibujos e improntas y luego, cada vez mayor número de fotografías. Fita compuso con esos datos sus Noticias, Informes y Viajes epigráficos, pero no siempre reprodujo completamente y al pie de la letra lo recibido, especialmente en el caso del material gráfico, por obvias dificultades técnicas y económicas. Esa correspondencia es también útil por cuanto ofrece un cándido testimonio del método de trabajo del ilustre académico ${ }^{12}$ e identifica a sus informantes, quienes, dejando a un lado los personajes de relumbrón, fueron en su mayoría gente de la que ape-

11 Hasta los años 90, el único repositorio del que se tenía noticia eran los legajos del Archivo de la Academia de la Historia, que llamaban la atención por la escasa documentación que contenían, vid. ABASCAL PALAZÓN, Juan Manuel, «Fidel Fita y la epigrafía hispano-romana», Boletín de la Real Academia de la Historia, 193, (1996), 305-334. A partir de entonces, se abrió la posibilidad de catalogar y consultar la mucho más numerosa serie custodiada en el Archivo Histórico de la Provincia de Castilla, de la Compañía de Jesús, en Alcalá de Henares, cuyo aprovechamiento resulta muy fructífero, vid. GARCÍA IGLESIAS, Luis, «Expansiones de académico a académico: Una carta del Marqués de Monsalud al P. Fita Colomer, S.J.», Revista de Estudios Extremeños, 50, (1994), pág. 566-612 y GÓMEZPANTOJA, Joaquín Luis, «El P. Fita y sus fuentes», Signo, 3, 1996, pag. 125-131. Con posterioridad, una pequeña parte de esos fondos, digitalizados, se han abierto a la consulta pública a través de http://bib.cervantesvirtual.com/portal/archivo_jesuitas/.

12 No fue infrecuente que Fita corrigiera o enmendara las informaciones recibidas de sus correspondientes, lo que comúnmente le llevó al yerro: ahora, cuando se redescubren los monumentos originales, se da la paradoja de que las transcripciones de los legos resultan mucho más perfectas que las del experto , vid, GÓMEZ-PANTOJA, Joaquín Luis, «Experto credite. El P. Fita y el anticuarismo soriano», en MORA, Gloria y DÍAZ-ANDREU, Margarita (eds.) La cristalización del pasado: Génesis y desarrollo del marco institucional de la Arqueología en España, Málaga, 1997, pág. 197-205. 
nas se conoce su nombre, el lugar de origen o residencia y, a veces, algún otro dato bio-bibliográfico, como la profesión ${ }^{13}$.

Este artículo trata de la asimétrica correspondencia mantenida con Agustín Sánchez Rodrigo, a propósito de algunas inscripciones antiguas encontradas en Serradilla, y en lugares vecinos de la provincia de Cáceres. La relación epistolar se inició después que Sánchez Rodrigo remitiese a la Academia de la Historia un ejemplar de la cuarta entrega de El Cronista, la publicación quincenal que había fundado un par de meses antes y que difundía por toda España entre los miembros de un influyente sector profesional; en ese número, fechado el 20 de febrero de 1916, el propio Sánchez había escrito una breve nota referida, entre otras informaciones, a un epitafio antiguo hallado en el pueblo y del que proporcionaba un facsímil, sin interpretarlo por falta de suficiencia ${ }^{14}$. Esta nota llamó tanto la atención de Fita que la reprodujo íntegra en una Noticia datada cinco días después y que tituló «Nueva lápida romana de la Serradilla»; como él sí tenía suficiencia, la segunda parte de la Noticia ofrecía una lectura más perfecta de la lápida basada en el apunte de Sánchez Rodrigo (vid. infra 3.1) ${ }^{15}$.

Estando aún en pruebas el Boletín, Fita envió a Serradilla los correspondientes folios y el día 8 de marzo, Agustín Sánchez le respondió agradeciéndole el interés y la diligencia, poniéndose a su disposición y ofreciéndose a complementar lo ya publicado con una foto de la pieza. El P. Fita contestó tres días después y aunque se desconoce el tenor exacto de la misiva, sí que sabemos algo de su contenido porque en el número sexto de El Cronista (20 de marzo de 1916), Sánchez Rodrigo dio noticia de lo publicado en el Boletín académico y su intercambio epistolar con el jesuita, quien le había encargado algunas averiguaciones sobre los epígrafes encontrados en Casas de Millán, Mirabel y Cañaveral, que son lugares más o menos vecinos de Serradilla. A fines de ese mes, otra carta desde Serradilla hizo llegar a Madrid la foto de la inscripción ya conocida, otra de un bloque granítico en el que se ven dos bustos (vid. infra 3.2) y un dibujo de otro epígrafe hallado en la finca «La Burra», con su texto y traducción (vid. infra 3.3).

Indirectamente consta que Fita contestó a ese envío pero Agustín Sánchez no volvió a escribir al P. Fita hasta el 20 de diciembre, indicándole que no había podido desplazarse a la dehesa «La Burra» para hacer la fotografía de la inscripción allí encontrada, pero notificaba el hallazgo de otra nueva en «La Herrera», de la que sí envió fotografía (vid. infra 3. 4); además adjuntaba a la carta una lista numerada — «para sin confusión referirnos mutuamente a ellas»- de las inscripciones encontradas hasta ese momento en Serradilla y alrededores. Fita ya no respondió a esa comunicación y

13 Una lista no exhaustiva de los informantes de Fita, pero limitada a los artículos publicados en el Boletin y a la correspondencia del jesuita existente en el Archivo de la Academia, en ABASCAL PALAZÓN, J.M., Op. cit. 1999, pág. 110 y ss.

14 SÁNCHEZ RODRIGO, Agustín, «Historia. Antigüedades de Serradilla», El Cronista, 1(4), 20 de febrero de 1916, s.p.

${ }_{15}$ FITA, Fidel, «Nueva lápida romana de La Serradilla», Boletín de la Real Academia de la Historia, 68, 1916, pag. 282-284. 
Sánchez se dirigió por última vez al académico con fecha de 24 de mayo de 1917, reprochándole cortésmente el silencio, porque «transcurre el tiempo (ya bastante) y nada dice $\mathrm{V}$. he pensado pudiera haberse extraviado la carta mía».

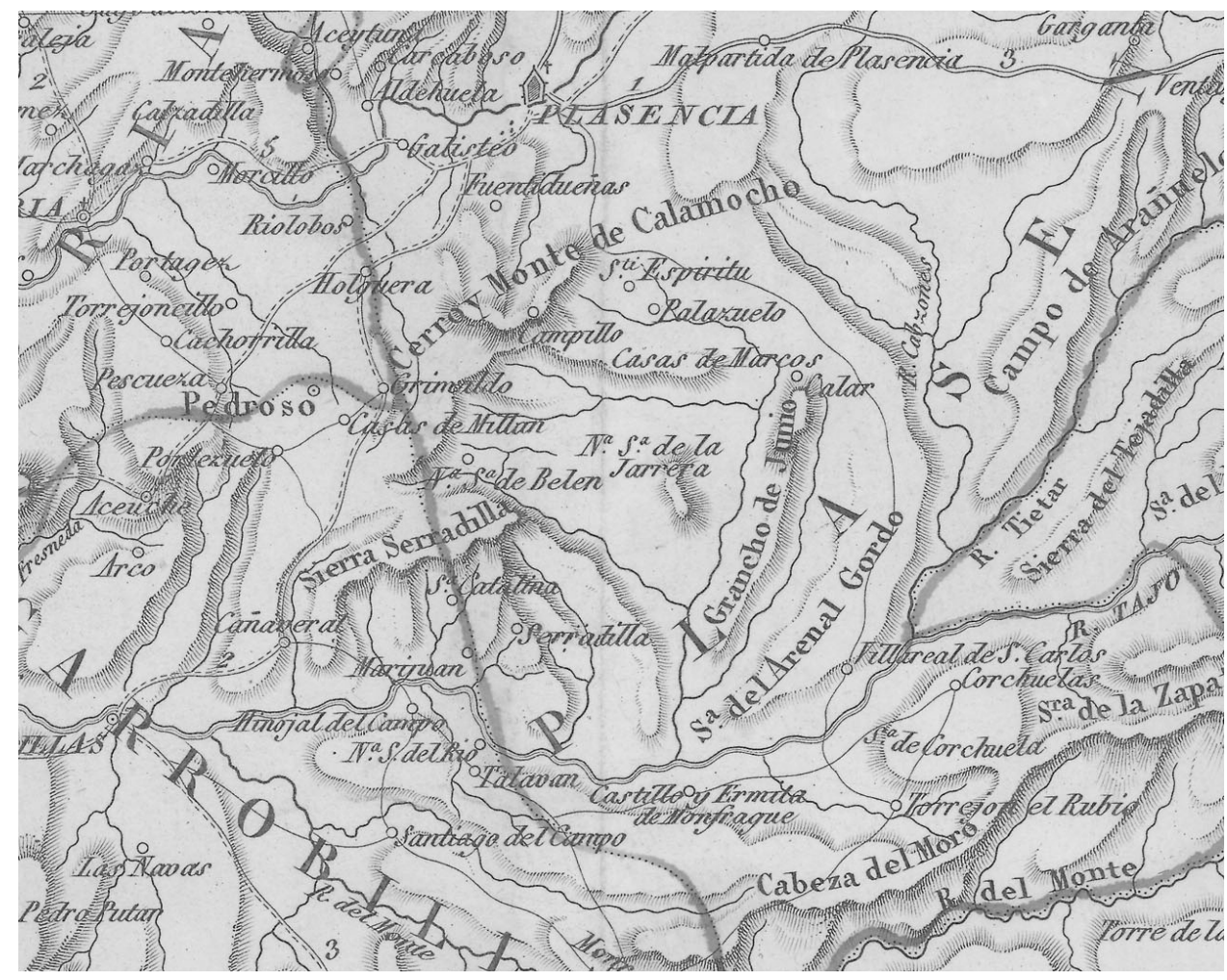

Fig. 1: Serradilla y sus alrededores según un fragmento del Mapa de la provincia de Cáceres grabado por R. Alabern y E Moban, 1846. En el centro del mismo se ve Serradilla, al pie de la Sierra del mismo nombre; justo al norte del ésta, se encuentra la Jarrera en la jurisdicción de Malpartida de Plasencia. Al sureste de Serradilla y en la orilla meridional del Tajo, están Torrejón El Rubio, emplazado en la solana de la Sierra de la Corchuela y más al este, Las Corchuelas. Reproducido con permiso de Instituto Cartográfico de Cataluña.

Ya muerto Fita, El Cronista de junio de 1918 publicaba una sencilla y breve relación de las cinco inscripciones encontradas en meses anteriores y de las que no se había dado noticia antes porque se estaba a expensas de un dictamen del ilustre académico que nunca llegó ${ }^{16}$. Por lo que sabemos, El Cronista y su editor no

16 SÁNCHEZ RODRIGO, Agustín, «Notas históricas. Antigüedades de Serradilla», El Cronista, 3 (60), 20 de junio de 1918, s.p. De las cinco inscripciones, sólo cuatro son antiguas, porque la última, un «ladrillo de barro con leyenda cristiana, hallado en las proximidades de la Plaza donde existió un antiguo templo; mide de ancho $12 \mathrm{~cm}$ y de largo 25», parece novicia. 
volvieron a ocuparse de temas epigráficos, salvo por una nota en el número de agosto del mismo año que refería el hallazgo de una nueva inscripción en el paraje de Las Corchuelas, jurisdicción de Torrejón El Rubio, vid. infra 3.5 ${ }^{17}$.

\section{LAS INSCRIPCIONES}

La correspondencia con Fita perjudicó a Sánchez Rodrigo por cuanto le obligó a retrasar la publicación de noticias en El Cronista, a la espera del dictamen del docto académico; pero también le convirtió en víctima de una pequeña injusticia historiográfica, ya que, con excepción de la primera lápida reproducida por Fita, las otras publicadas por él pasaron desapercibidas en la literatura científica hasta que la apertura de los «papeles Fita» del Archivo Histórico jesuítico de Alcalá de Henares han puesto al descubierto las fotografías enviadas a la Academia de la Historia. El valor de ese testimonio gráfico es fácil de comprender por cuanto las piezas fotografiadas no se conocían hasta ahora y, además, se dan por perdidas; de ahí que recientemente se hayan editado siete epígrafes extraídos de la correspondencia de Antonio (sic!) Sánchez Rodrigo y de otro informante extremeño de Fita, Juan Sanguino y Michel, entre ellos cuatro de los mencionados antes ${ }^{18}$. Pero al llevar a cabo la actualización de los datos de la base de datos «Hispania Epigraphica Online» descubrimos entre los «papeles Fita» otro datos relativos a Serradilla no tenidos en cuenta por Salamanqués ${ }^{19} \mathrm{y}$, sobre todo, se despertó nuestro interés por El Cronista como fuente de información bibliográfica. Lo que ahora ofrecemos es una más completa información sobre cinco epígrafes descubiertos en Serradilla y sus alrededores y casi todos ellos publicados por primera vez por Sánchez Rodrigo entre 1916 y 1918.

\section{Serradilla, el Rincón de Palermo}

«Lápida funeraria, cuya inscripción aún inédita es como sigue....

Se encuentra esta lápida incrustada en la pared de una casa en el Rincón de Palermo [...]. A esta circunstancia de haberla colocado en la pared quizá se deba su conservación, pues si no, dada la escasez que hay aquí de la piedra de granito,

17 SÁNCHEZ RODRIGO, Agustín, «Otra lápida romana», El Cronista, 3 (64), 20 de agosto de 1918, s.p.

18 SALAMANQUÉS PÉREZ, Virginia, «Nuevos datos para la epigrafía de la provincia de Cáceres en la correspondencia de Fidel Fita con Antonio Sánchez y Juan Sanguino y Michel», en VENTURA, Ángel y MATEOS CRUZ, Pedro (ed.), Espacios, usos y formas de la epigrafía Hispana en épocas Antigua y Tardoantigua: homenaje al Dr. Armin U. Stylow, Mérida - Madrid, 2009, pág. 323-330.

19 Por ejemplo, que el existencia de inscripciones antiguas en Serradilla no debía de ser sorpresa para el P. Fita, porque el propio Sanguino, secretario entonces de la Comisión de Monumentos de Cáceres, le comunicó por carta a fines de junio de 1905 que «de Serradilla me anuncian también otra inscripción y pido calco», vid. http://bib.cervantesvirtual.com/servlet/SirveObras/90259519876025376410457/p0000003.htm. 
ya hubiera sido aplicada a cualquier fin sin reparar en inscripciones más o menos viejas. En cuanto a la interpretación de la inscripción completando las faltas de letras que hay en las líneas $3 .^{-\underline{a}}, 4 .^{\underline{a}}, 7 .^{\underline{a}}$ y última, es labor para la cual no me considero suficiente. Ya lo hará quien para ello tenga competencia»²0.

\section{P E T R O N I A}

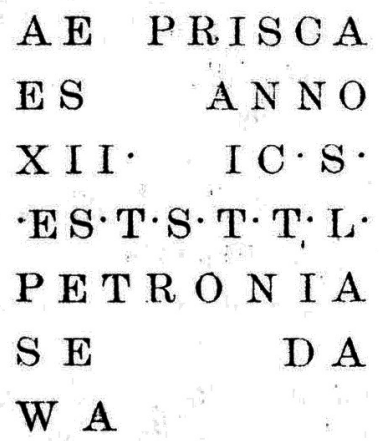

Fig. 2: Copia de la inscripción del Rincón de Palermo, en Serradilla, según Sánchez Rodrigo.

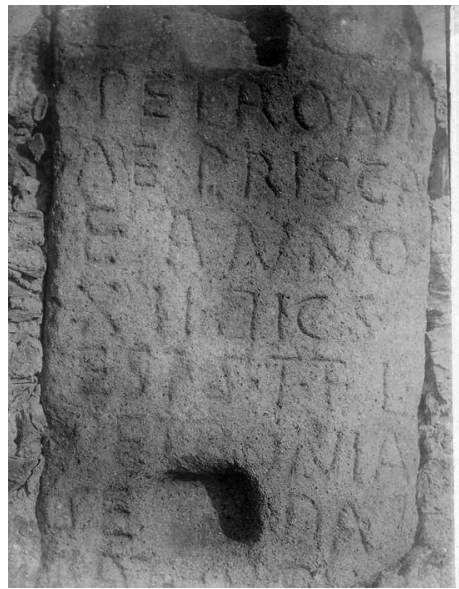

Fig. 3: Fotografía de la inscripción del Rincón de Palermo, en Serradilla, remitida por A. Sánchez Rodrigo a Fita en 1916. Archivo Histórico de la Provincia de CastiIla, Alcalá de Henares

A lo anterior, Sánchez Rodrigo añadió la foto de la pieza y también el dato de que se encontraba «sita en la fachada de la casa de Félix Fernández en el Rincón de Palermo[...]. Su tamaño es alto - $71 \mathrm{~cm}$ - y ancho - $40 \mathrm{~cm}$ ».

Bloque de granito mutilado por arriba y por abajo. Por una reutilización posterior tiene dos orificios rectangulares; uno de ellos, en la línea 7, que afecta ligeramente al texto. Medidas: (71) $\times 40 \times$ ? cm. Letras: c. $5 \mathrm{~cm}$; capital cuadrada con rasgos rústicos. Interpunción: punto redondo. Estuvo en la fachada de la casa de Félix Fernández, en el Rincón de Palermo, en Serradilla. En nuestra opinión, se trata muy posiblemente del epígrafe cuyo hallazgo Sanguino anunció por carta a Fita en 1905, vid. supra nota 19; como en ese año, Sánchez Rodrigo estaba ya muy ocupado con la edición del Rayas, resulta explicable que no enviara más información a Sanguino. Dado que la inscripción está en paradero desconocido, la leemos a partir de los datos anteriores y de la foto citada:

SÁNCHEZ RODRIGO, A. 1916, op. cit., s.p. FITA, F. 1916, op. cit. pág. 282284; HURTADO DE SAN ANTONIO, R., Corpus provincial de inscripciones latinas:

20 SÁNCHEZ RODRIGO, A., op. cit., 1916, s.p. 
Cáceres, Cáceres, Diputación Provincial de Cáceres, Servicios Culturales, 1977, cat. n. 468; SALAMANQUÉS, V., op. cit. 2009, pág. 326-7; HEpOI 15083.

Petroni-

ae Prisca-

e anno(rum)

$X I I \cdot h i c \cdot s(i t a) \cdot$

5 est $\cdot s($ it $) \cdot t($ ibi $) \cdot t($ erra $) \cdot I($ evis $)$

Petronia

Se[cun]da

mater su[a]

- - - - .

2: S(exti) [f(iliae)], Fita, debido a que en el apógrafo publicado en El Cronista aparecía una S y Sánchez Rodrigo afirmaba la ausencia de letras en ese renglón.

4: En la fotografía, detrás del numeral XII, parece verse un pequeño trazo vertical en el nivel inferior del renglón, e, inmediatamente, un trazo vertical del que a su izquierda surge, en la parte superior, otro trazo horizontal, parecido a una L rotada en $180^{\circ}$.

8: ma[ter f(aciendum) c(uravit)] Fita; $d(e) s(u o)$, Salamanqués. En la foto, al final del r. se aprecian el arco superior de la $S$ y los trazos finales superiores de la V. La gens Petronia está bien representada en la epigrafía cacereña ${ }^{21}$. Otra Petronia Prisca está atestiguada en Esparragosa de La Serena, provincia de Badajoz ${ }^{22}$. Tanto Prisca como Secunda son frecuentes entre los cognomina hispanos.

\section{Serradilla, El Rincón de San Pedro}

«Está situada a la altura de un metro próximamente (sic) en la fachada de la casa del vecino de esta villa Doroteo Barbero Cobos (sic!), en el Rincón de S. Pedro. Es de granito, siendo el tamaño de la superficie que presenta al exterior, de 50 x 56 centímetros. Aunque bastante deteriorados, se aprecian bien en esta piedra

21 Con cierta concentración en los alrededores de Trujillo: Petronia Marcella en el mismo Trujillo (AE 1977, 404; HEpOI 20327); P. Petronius Firmanus en Ibahernando, vid. REDONDO RODRÍGUEZ, JOSÉ A. y ESTEBAN ORTEGA, Julio «Estelas funerarias de Ibahernando (Cáceres)», Ficheiro Epigráfico, 96, 2012, 429; Petro[- - -] Sever[- - - ] en Santa Marta de Magasca, Inédita, datos de J. Esteban; Petronia Secundina en Salvatierra de Santiago (HEp 16, 2007, 117; HEpOI 19389); Lucius Petronius Severus en Puerto de Santa Cruz (CPILC 405; HEpOL 23067; Petronius Capito y Severus en Cáparra (HEp 11, 2001, 124; HEpOI 24333) y L. Petronius Niger, Ilvir, en Cáceres (CIL II 693; AE 1919, 88; HEpOI 21671).

${ }^{22} \mathrm{CIL} \mathrm{I} 2 / 7,941 ; \mathrm{HEpOI} 4514$. 
dos bustos: uno de hembra a la izquierda y otro de varón a la derecha del observador. No se ven resquicios de inscripción alguna. Mandé fotografía al P. Fita, quien me contestó expresando su opinión de que la lápida era del siglo II o III del Imperio» 23 .

Fragmento superior de un monumento funerario de granito, que por la anchura posiblemente no fuera una estela. Se aprecian, en bajorrelieve, dos bustos humanos y no existen restos de inscripción, si es que la tuvo. Medidas: (50) x (56) $\mathrm{x}$ ? cm. Estaba incrustada, a un metro de altura, en la fachada de una casa de la calle Rincón de San Pedro, propiedad de Doroteo Cobos Barbero ${ }^{24}$.

SÁNCHEZ RODRIGO, A., op. cit. 1918, cat. n. 2; SALAMANQUÉS, V. op. cit. 2009, pág. 323-30, como inédita.

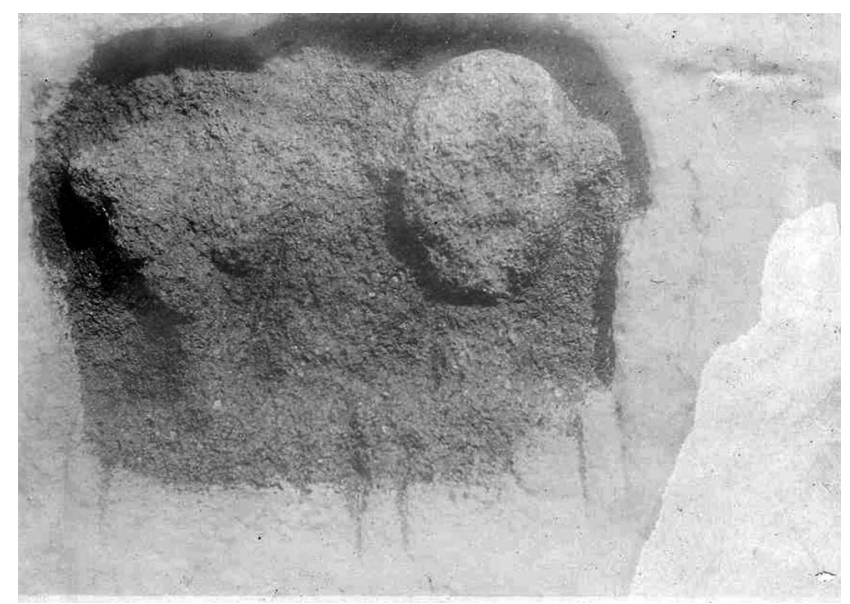

Fig. 4: Fotografía del relieve funerario del Rincón de San Pedro, en Serradilla, remitida por A. Sánchez Rodrigo a Fita en 1916.

Archivo Histórico de la Provincia de Castilla, Alcalá de Henares.

En un radio aproximado de $20 \mathrm{~km}$ entorno a Serradilla están atestiguados otros dos bajorrelieves: el primero es una hornacina con busto humano en un monumento funerario encontrado en la finca San Rafael, en Torrejón el Rubio. El segundo es un fragmento de la parte superior de una estela funeraria, con dos figuras humanas, aparecida en Monroy ${ }^{25}$.

23 SÁNCHEZ RODRIGO, A, op. cit. n. 2, 1918; cf. cartas de A. Sánchez a F. Fita, de 29/03/1916 (con fotografía) y 20/12/1916, Archivo Histórico de la Provincia de Castilla de la Compañía de Jesús, Alcalá de Henares.

24 Sánchez Rodrigo alterna los apellidos del propietario de la casa donde se encontraba el bajorrelieve. Consultada la vigente guía telefónica, un miembro de la familia Cobos sigue siendo titular de la línea telefónica de la casa del Rincón de San Pedro.

25 Respectivamente HEp 3, 1993, 140; AE 1991, 977; HEpOL 22741 y ESTEBAN ORTEGA, Julio y SALAS MARTÍN, José, Epigrafía romana y cristiana del Museo de Cáceres, Cáceres, Museo de Cáceres, Consejería de Cultura, Junta de Extremadura, 2003, cat. n. 95. 


\section{Torrejón el Rubio - Dehesa La Burra}

«Hallada en la dehesa "La Burrilla"26, muy próxima a la carretera de Plasencia a Trujillo, en las inmediaciones del río Almonte. Hoy está en la casa que tienen los dueños en referida dehesa. Sus dimensiones, 85 x 35 centímetros. En la parte superior tiene un sol con seis radios, de 30 centímetros de diámetro, y la siguiente inscripción:

\section{S E V E R A R F U F. A N VII USES T. T. L.}

Fig. 5: Apógrafo de la inscripción de la dehesa La Burra en Serradilla, según Sánchez Rodrigo.

Cuando di al P. Fita la primera noticia de esta lápida, sólo le enviaba copia de la inscripción, prometiéndole fotografía y más detalles. Cuando me contestó dice: "La inscripción se deja bien leer y entender, menos el número de años (¿XVII?) de la difunta Severa, hija de Rufo"»27. Por carta a Fita, Sánchez Rodrigo añadió otros detalles de interés: que en la zona del hallazgo «hay allí numerosos y grandes villares, a uno de los cuales se le llama "La Villeta" que hay un vado en el Almonte defendido por una trinchera abierta en la roca»28.

Igualmente, la copia de la inscripción enviada a Fita es un apunte obtenido por quienes hallaron la lápida y Sánchez Rodrigo anotó que «no respondo de la colocación de las letras ni de su exactitud». Al parecer, no llegó a ir al lugar para hacer la foto que tenía anunciada ${ }^{29}$.

Estela de granito, posiblemente con remate semicircular decorado con una rosácea hexapétala inscrita en un doble círculo. Medidas: (85)? x 35 x ?. Interpunción: redonda. Se halló en la Dehesa La Burra y se conservaba en la casa de la misma finca.

${ }^{26}$ En su correspondencia con Fita siempre se refiere al lugar del hallazgo como «La Burra»; ambas fincas son colindantes, pero la más cercana al Almonte es ésta.

27 SÁNCHEZ RODRIGO, A. op. cit. 1918.

28 Sin duda el castro de las Villasviejas del Azuquén de la Villeta, distante unos $14 \mathrm{~km}$ al Oeste del caserío de la Burra, que está situado en la confluencia de los ríos Tozo y Almonte; en el flanco del despoblado no protegido por los cauces fluviales, la defensa se confió a un amplio y profundo foso, vid. http://www.asociacionelbezudo.com/villaromana/enlacesvilla/rastreo.htm.

29 Cartas de A. Sánchez a Fidel Fita de 29/03/1916 y 20/12/1916, Archivo Histórico de la Provincia de Castilla de la Compañía de Jesús, Alcalá de Henares. 
SÁNCHEZ RODRIGO, A. op. cit. 1918, cat. n. 3; SALAMANQUÉS, V., op. cit. 2009, págs. 324-325, como inédita.

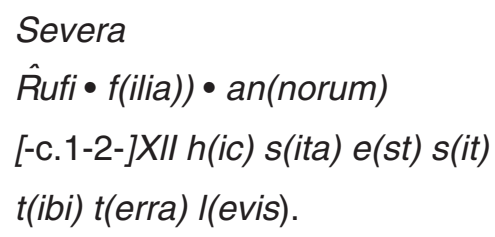

La decoración con hexapétala está muy extendida por toda la comarca, siendo también abundantes las inscritas en doble círculo. La onomástica es banal porque Severus/a es, según Abascal ${ }^{30}$ el más corriente de los cognomina usados en Hispania, seguido de Rufus/a.

\section{Malpartida de Plasencia - Dehesa Herrera o La Jarrera}

«Encontrada en la dehesa Herrera, sobre el camino de Serradilla a Plasencia, término municipal de Malpartida de Plasencia. Es de granito, y las dimensiones de su superficie son 32 × 27 centímetros. Esta piedra debe considerarse como fragmento solamente, por su lastimoso deterioro. Por las partes superior e inferior, se ven líneas partidas que no pueden leerse, y el centro tiene una oquedad producida por haber servido de quicio a una puerta o angarilla, quizá durante siglos y siglos. Las letras que regularmente se entienden son las siguientes: ${ }^{31}$

\section{E P. VRR V \\ P. $\mathrm{H} \quad \mathrm{R} \mathrm{VE}$ \\ $\mathrm{M} \quad \mathrm{E} \mathrm{N}$}

\section{$\mathrm{A} \perp \mathrm{E} \quad \mathrm{E} \mathrm{E}$}

Fig. 6: Apógrafo del fragmento de "La Herrera", en Malpartida de Plasencia, según Sánchez Rodrigo

30 ABASCAL PALAZÓN, Juan Manuel, Los nombres personales en las inscripciones latinas de Hispania, Murcia, Universidad, 1994, pag. 506 y 487.

31 SÁNCHEZ RODRIGO, A., op. cit. 1918, cat. n. 4. Cf. Carta de A. Sánchez a F. Fita del 20/12/1916, con fotografía, Archivo Histórico de la Provincia de Castilla de la Compañía de Jesús, Alcalá de Henares 


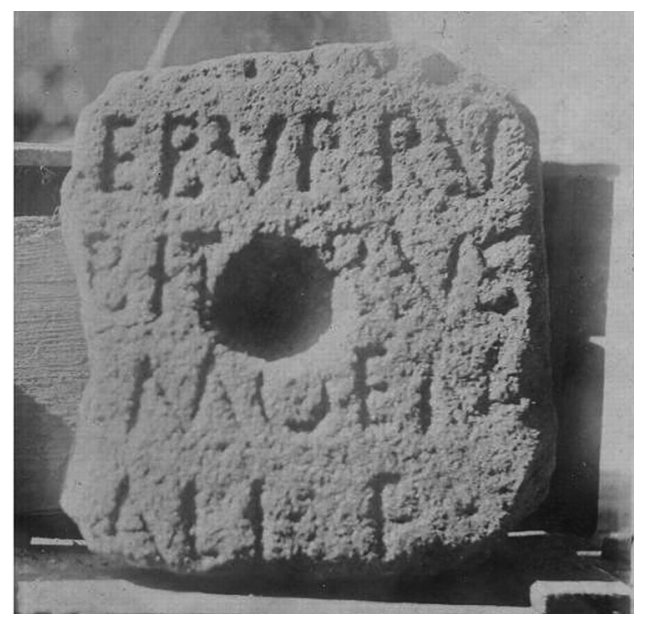

Fig. 7: Fotografía del fragmento de "La Herrea" remitido al P. Fita por Sánchez Rodrigo. Archivo Histórico de la Provincia de Castilla de la Compañia de Jesús, Alcalá de Henares.

Fragmento cuadrangular de granito perteneciente a la parte central de una lápida; en el centro tiene un rebaje circular debido a su uso como quicialera. Medidas: (32) x (27) x ?. Letras: c. 4,5; capital rústica. Interpunción: puntos redondos. Se halló en la finca La Herrera, que «la cruza la Vereda del Rey de E. a O. y el camino de Serradilla a Plasencia de S. a N».

SÁNCHEZ RODRIGO, A. op. cit. 1918, cat. n. 4; SALAMANQUÉS, V., op. cit. 2009, pag. 325-326, como inédita.

$[---]+++[---]$

[R]eburrûs

[Do]bit[e]rus

[A]moen̂a

5 [C]ali $\cdot f($ ilii) .

Hay restos inidentificables de letras en r. $1-5$ : [Cam]ali, Salamanqués, pero la restitución no respeta el número de letras que faltan por la izquierda.

Como puede verse, la inscripción presenta una relación de nombres en nominativo y, en el último renglón, el patronímico; posiblemente se trata de los dedicantes del epitafio, estando expresado el nombre del difunto en la parte perdida de la lápida. Reburrus es uno de los más corrientes nombres no latinos de Hispania, 
pero en Cáceres solo está atestiguado en dos ocasiones, en una inscripción de Talavera la Vieja y en otra, algo dudosa, de Baños de Montemayor ${ }^{32}$; Dobiterus, por el contrario, está ampliamente representado en la comarca, al igual que Amoena; finalmene, Calus también aparece en varias ocasiones en epígrafes cacereños ${ }^{33}$.

Debe notarse que éste no es el único hallazgo de la «La Herrera» 0 «La Jarrera», porque hay noticias de la aparición en ese término de dos inscripciones. De la primera dio noticia J. Sanguino y Michel al P. Fita en una carta enviada desde Santoña el 18 de noviembre de $1910^{34}$ y se trataba de un fragmento de granito reutilizado como dovela y que aún conservaba tres líneas de texto pertenecientes, quizá, a una dedicatoria a Júpiter ${ }^{35}$. Y la segunda se refiere a «un pedestal con inscripción», de cuyo hallazgo, sin más datos, se dio cuenta a la Comisión de Monumentos Históricos y Artísticos de la provincia de Cáceres el 22 de marzo de $1920^{36}$.

\section{Torrejón el Rubio, Las Corchuelas}

«En la próxima dehesa Las Corchuelas, propiedad del Exmo. Sr . Conde de Trespalacios, varios obreros que estaban descuajando hallaron una losa de pizarra que tenía muchas letras grabadas. Por mediación de un hijo del guarda de aquella parte de la finca, he obtenido una copia que él mismo ha hecho de la inscripción y es como sigue:

$$
\begin{aligned}
& \text { N V I A } \cdot \text { C } \cdot \text { F }
\end{aligned}
$$

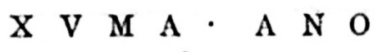

$$
\begin{aligned}
& \text { A . V . H } \cdot \mathrm{S} \cdot \mathrm{T} \cdot \mathrm{T} \mathrm{L}
\end{aligned}
$$

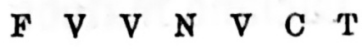

$$
\begin{aligned}
& \text { F C }
\end{aligned}
$$

Fig. 8: Apógrafo de la inscripción de "Las Corchuelas", en Torrejón el Rubio según Sánchez Rodrigo.

\footnotetext{
32 Respectivamente, CIL II 5343 = HEp 10, 2000, 141 = HEpOL 6463; y CIL II 886 = HEpOL 21824

${ }_{33}$ Alcuéscar: HEp 6, 1996, 193; AE 1998, 719; HEpOL 23279); Valdefuentes: HEp 15, 2006, 100; AE 2006, 623; HEpOL 25617); y Villamesías: HEp 4, 1994, 257; AE 1991, 979; HEpOL 23073.

34 Sanguino a Fita. vid. http://bib.cervantesvirtual.com/servlet/SirveObras/9025951987602537 6410457/index.htm. La editio princeps del hallazgo en BERJANO ESCOBAR, Daniel, »Notas epigráficas». Revista de Extremadura 11, 1909, págs. 574-575.

${ }_{35}$ ALBERTOS FIRMAT, María Lourdes, «Nuevas divinidades de la Antigua Hispania», Zephyrus, 3, 1952, p. 61. MELENA, José Luis, «Salama, Jalama y la epigrafía latina del antiguo corregimiento», en Symbolae Ludovico Mitxelena septuagenario oblatae, pars prior, Vitoria, 1985, pág. 475-530; HEpOI 24938,

${ }^{36}$ HURTADO, Gustavo, «Comisión de Monumentos Históricos y Artísticos de la provincia de Cáceres» [Sesión 22/03/1920]», BRAH77, 1920, pag. 91.
} 
No puedo hoy dar las medidas del tamaño de la piedra ni garantir (sic!) la exactitud de la inscripción, limitándome a dar noticia de su existencia, pero prometo no tardar mucho en obtener una fotografía que sea base sólida para su estudio e interpretación » ${ }^{37}$.

Losa de pizarra, rota por la izquierda. En la interpunción hay que suponer que se utilizaron puntos redondos. La Dehesa de las Corchuelas está situada en la falda norte de la Sierra de Monfragüe y cerca de la embocadura del llamado «Puente del Cardenal», en la margen meridional del río Tajo ${ }^{38}$.

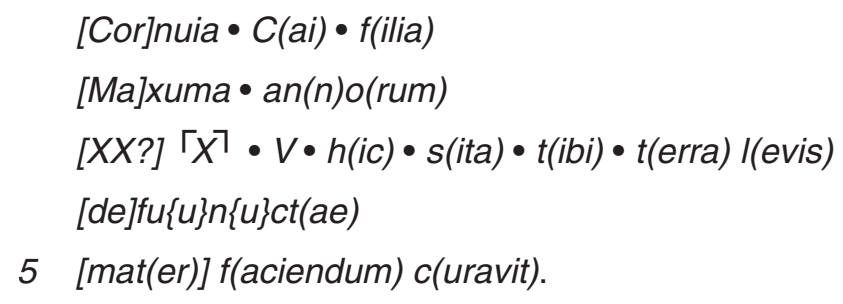

El informante de Agustín Sánchez Rodrigo no parece muy versado en letras y por ello, debe considerarse el dibujo proporcionado más como un apunte que una copia, lo que añade mayor dificultad a la interpretación del epígrafe. Un punto de partida seguro es el cognomen de la difunta, que permite suponer que se han perdido dos o tres letras al inicio de cada línea.

1: Son raros los nomina femeninos terminados en -nuia ${ }^{39}$; en razón del espacio, el que más conveniente es Cornuia, sólo constatado en Etruria ${ }^{40}$ - 3: La fórmula de deposición omitiendo ambos verbos, está atestiguada en un par de epitafios de la provincia ${ }^{41}$. Las dificultades están en el inicio del r., donde se podría entender que la A fuera la parte derecha de una M, obteniéndose an(n)o/[ru] $\lceil m\rceil$,

37 SÁNCHEZ RODRIGO, A., op. cit. 1918. De la noticia de El Cronista. tuvo eco la Comisión de Monumentos de Cáceres por intermedio de Juan Sanguino, quien se abstuvo de juzgar el hallazgo hasta disponer de la prometida fotografía, vid, HURTADO, Gustavo, «Comisión Provincial de Monumentos Históricos y Artísticos de Cáceres [Actas de las sesión del 22 de octubre de 1918]», BRAH 74, 1919, p. 270.

38 Esta imponente obra de ingeniería civil fue mandada construir por el obispo de Plasencia y Cardenal de la Iglesia de Roma Juan de Carvajal (1446-1469), se encuentra a unos diez km de distancia de Serradilla, aguas arribas del Tajo y sirve de límite jurisdiccional entre esa población y Torrejón el Rubio; a mediados de los años 70 quedó anegada por la cola del embalse de Alcántara, pero desde su construcción hasta la expansión del ferrocarril, fue uno de los cinco vados artificiales del Tajo en el tramo de más de $500 \mathrm{~km}$ de río desde Lisboa a Toledo; los otros fueron los puentes de Alcántara y Almaraz, en Cáceres; y los del Arzobispo y, de nuevo, de Alcántara, en Toledo. Sobre sus características y vicisitudes históricas, vid. MACHIMBARRENA, Vicente, «Puentes sobre el río Tajo (III). Provincia de Cáceres. Puentes del Cardenal, Almaraz, Alarza y Conde», Revista de Obras Públicas, 74, 1926, pág. 521-524.

${ }^{39}$ El más corriente Ingenuia, con media docena de testimonios, mientras que Cornuia y Utronuia se evidencian una sola vez.

40 CIL XI 2669: - - - - - / [Te]rtulla / vix(it) a(nnos) II m(enses) VII / Cornuia Vitalis / mater pos(uit).

41 En Ibahernando (REDONDO RODRÍGUEZ, José A., Catálogo epigráfico-latino de Trujillo y su partido judicial, Memoria de Licenciatura de la Universidad de Extremadura, Cáceres 1983, cat. n 1) y en Salvatierra de Santiago (HEp 16, 2007, 117; HEpOI 19389). 
una indicación poco usual por no ir abreviada, mientras que la alternativa a(ve!) $v(a l e !)$, además de ser inédita en Cáceres, no parece adecuada en el contexto rural en que se halló la inscripción. Por eso, es más fácil suponer que se trata de una $\mathrm{X}$ mal leída, y posiblemente faltarían uno o dos numerales más de la edad 4: En Extremadura, el adjetivo defunct- aparece en Montijo ${ }^{42}$ y, con su sigla, en Talavera la Vieja $d$ (efuncto) ${ }^{43}$; Probablemente también en una inscripción inédita de Zalamea de La Serena y en Capera, según la foto publicada por Mélida ${ }^{44}$. Cabe en lo posible que esas letras correspondan al nombre de la dedicante - 5: mat(er) es la solución banal y en consonancia con lo habitual en las lápidas de la zona.

Nótese que éste no es el único hallazgo epigráfico producido en la zona, ya que Ponz, durante su famoso Viaje, vio una estela de granito de grandes dimensiones y cabecera redondeada empotrada en el mencionado puente del Cardenal ${ }^{45}$; puede sorprender que un monumento de más de un metro de alto le pasase desapercibido a Sánchez Rodrigo, pero lo cierto es que tampoco Hübner lo anotó y hubo que esperar a 1964 para que volviera a ser redescubierto ${ }^{46}$.

\section{A MODO DE CONCLUSIÓN}

En el prefacio de The Roman Revolution, Syme recordaba que el historiador puede y debe usar las evidencias de las que dispone, no tanto por su irrecusable libertad intelectual cuanto porque la Historia es una ciencia imperfecta y queda mucho por hacer. Este artículo ha tratado de seguir tal precepto, añadiendo a la cambiante historia del progreso de la Epigrafía en Hispania la modesta aportación de lo que un poco conocido dilettante aldeano hizo para que nos se perdieran los más remotos testimonios escritos de la historia de su pueblo. Su proceder fue impecable ya que, consciente de su falta de suficiencia, puso en conocimiento del experto los nuevos datos; la fortuna y los achaques del Padre Fita quisieron que esa información permaneciera aparentemente oculta entre unos papeles manuscritos hasta hace poco años. Sin embargo, ello no debía haber importado porque las inscripciones fueron, a la postre, dadas a conocer por el propio Sánchez Rodrigo entre 1916 y 1918 de modo tan legítimo como para atribuirle justamente la primacía de la edición: los datos completos del hallazgo, lectura, lugar de conservación y una copia del texto conforme a lo acostumbrado en la época aparecieron publicados en un medio impreso con suficiente difusión. El que Fita primero y luego, la Co-

42 DURÁN RAMOS, Cristiana I., «Inscripción visigoda de Montijo», Habis 6, 1975, 365-368, lám XXXVII; HEpOL 25838.

43 CIL II 934; HEpOL 1797.

44 MÉLIDA, José Ramón, Catálogo Monumental de España. Provincia de Cáceres. (1914-1916), Madrid 1924, tomo III, fig. 63 (CIL II 839; HEpOI 21778).

45 PONZ, Antonio, Viage de España, vol. VIII, Madrid, Joaquín de Ibarra, 1784, pág. 155.

46 SÁNCHEZ PAREDES, Antonio, »Dieciséis inscripciones latinas, inéditas, de la Vettonia». Diario Extremadura, 07/02/1964, no 14 (HURTADO DE SAN ANTONIO, R., op. cit. cat. n. 750); cfr. HEpOI 22153. 
misión de Monumentos de Cáceres se hiciera eco de sendas noticias de esos hallazgos aparecidos en El Cronista tenía que haber servido de acicate para la perspicacia de quienes se han ocupado de la epigrafía cacereña y no debía haber sido necesaria la publicación de los «papeles Fita» para que las cinco inscripciones dadas conocer por Sánchez Rodrigo figurasen desde hace casi un siglo en los catálogos epigráficos provinciales. 